\title{
Research on Multicast Routing Algorithm for P2P Overlay Network
}

\author{
Chen Liangbin \\ Dept. of Aeronautic Ammunition Engineering \\ The First Aeronautics College of Air Force \\ Xinyang, China \\ liangbinchen2004@163.com
}

\author{
Li Qiang \\ Dept. of Aeronautic Ordnance Engineering Department \\ The First Aeronautics College of Air Force \\ Xinyang, China \\ 458306345@qq.com
}

\author{
Feng Xiang \\ Dept. of Aeronautic Ammunition Engineering Department \\ The First Aeronautic Institute of Air Force \\ Xinyang, China \\ wirelessfx@126.com
}

\begin{abstract}
Multicast routing is a critical problem in the P2P network. The main task for multicast routing is to establish a multicast tree with good performance, to satisfy the various qualities of service requirements, such as improving the streaming media service quality, and the utilization rate of cyber source. Many efficient overlay multicast routing algorithms are proposed to face the new challenge in current P2P network. This paper attempts to survey the current problems, the solutions for overlay multicast routing algorithms, and points out the further research directions.
\end{abstract}

Keywords-P2P; overlay network; multicast routing; algrithm

\section{INTRODUCTION}

The P2P streaming media system to the application surface continues to expand, which uses the terminal host bandwidth and computing ability to overcome the traditional $\mathrm{C} / \mathrm{S}$ distributed network, has been an independent media. Due to need of a lot of services and cyber source for largescale stream media, a lot of technology based on IP multicast has been appeared. Overlay multicast routing method is the one of the technologies.

Overlay multicast maintains the "unicast to best effort" model, mainly through the end system rather than the core router to realize multicast communication. It makes multicast group members to establish the data transmission of multicast tree directly and realizes corresponding function in the terminal host. In the network layer, the IP unicast data transmission is still used to eliminate the network infrastructure (such as multicast router) dependence.

Even Overlay multicast has the advantage of not need to change the existing IP network and can solve many problems, such as save the cyber source and reduce the network management requirements, but it still brings about some new problems. How to satisfy the application requirements of overlay multicast routing is one of the most important problems.

At present, overlay multicast technology has gradually replaced the IP multicast technology. But the overlay multicast network is built in IP network logic network, which has a worse routing characteristics than IP multicast, and which has become an obstacle to the development of the main problems in overlay multicast. Therefore, the multicast routing algorithm to meet the application needs of the QoS, a good scalability, adapted to the dynamic environment has become the research hotspot. In this paper, according to the multicast routing concepts are given in overlay multicast model, and discuss the typical routing algorithm and the existing problems, and points out the research direction in the future.

\section{RELATED CONCEPTS}

Overlay multicast was overcome some disadvantages on transmission technology and management. For example, a multicast group management overhead is large, poor reliability and safety. Overlay multicast is through the end system (application layer) rather than the core router to realize multicast communication in all functions, which includes group management, the group member management, packet replication and packet forwarding.

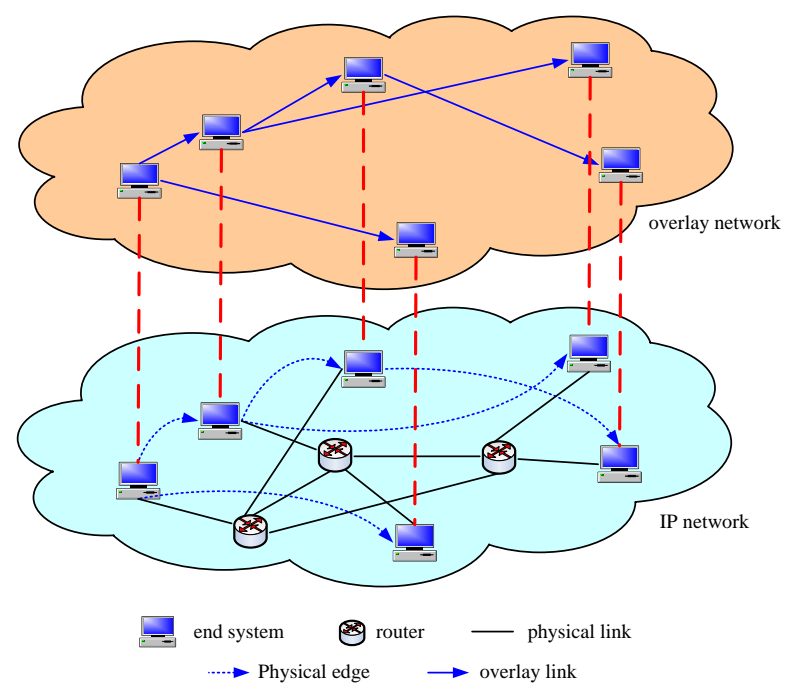

Figure 1. overlay multicast network 
The underlying IP network only needs to provide basic end-to-end unicast transmission service, so the overlay multicast known as application layer multicast. As Fig. 1 shows, the lower the IP network has end-to-end unicast communication function, and guarantees every overlay link correspond to the physical path communication.

In the overlay network, multicast function is by participating in the multicast group end system node to finish, end node forms overlay multicast tree through overlay link. Source node send data to the receiving node along the multicast tree, to each of non-leaf nodes receive data, and retransmit data to other child node data at the same time.

Problems of the overlay multicast network is built in IP network, overlay multicast are associated with the closely related multicast routing are a multicast routing is one of the many new multicast applications are concerned. Overlay multicast network model is put forward from the perspective of route optimization in this paper.

The presented overlay multicast network is constructed upon the IP network, if the underlying IP network to end system provides transparent end-to-end unicast routing, then overlay multicast network model can be used to complete directed graph to describe as Fig. 2.

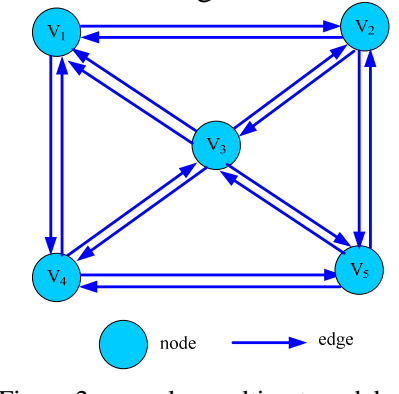

Figure 2. overlay multicast model

Fig. 2 gives the complete directed graph, named $G$. $G$ can be described in $G=(V, E), V$ is referred as the set of node, node refers to end system, $|V|=N, E$ is the set of edges, edges is referred as virtual link, $|E|=M$, a virtual link correspond to a physical path in the underlying IP networks. For $v \in V$, input bandwidth function $B_{\text {in }}(v)$ is referred as maximum bandwidth of node $v$, output bandwidth function $B_{\text {out }}(v)$ refers to maximum forwarding bandwidth of $v, C_{p}(V)$ is node price, such as the end of the cost of equipment system. For $e$ $\in E, e$ is edge which connected node $u$ to $v, e$ is refers to $(u, v), C_{1}(u, v)$ is refers as the maximum bandwidth that node $u$ and $v$ can achieve on the $e . C_{2}(u, v)$ is the end-to-end packet transmission delay between two nodes, $C_{3}(u, v)$ is refers to delay jitter end-to-end packet transmission, loss function $\operatorname{Cost}(e)$ is edge overhead.

According to the overlay multicast network model, overlay multicast routing problem can be described as follows: given a completely directed graph $G=\left(V^{\prime}, E^{\prime}\right), P$ is set on the path, the source node $S \in V$, and the destination node $T \in V$, find a path $p$ which is $S$ from to $T$, then $p \in P$, it make the cost minimum, and satisfy with constraint conditions. If there is $k$ constraint conditions, for a set of constraints $C$ and a possible optimization target $O$, construct an optimal spanning tree $G$, minimum $\sum_{(u, v) \in P} \operatorname{Cost}(u, v)$ to achieve optimum target $\mathrm{O}$, and to satisfy the constraint $\sum_{(u, v) \in P} C_{i}(u, v) \leq C_{i}^{\prime}$, which $C_{i}^{\prime}$ is constant, $C_{i}^{\prime} \in C, 1 \leq i \leq k$.

\section{Challenges In Overlay Multicast Routing}

Overlay multicast is more superior to IP multicast in the performance. It has three characteristics. Firstly, it can be deployed; overlay multicast only requires the underlying IP network supporting unicast communication function does not need to change the existing IP network infrastructure. The overlay multicast has the advantages in convenient deployment and lower cost. Secondly, it has the flexibility, overlay multicast can use terminal system with computing capability, and easy to achieve. Thirdly, it is extensible; overlay is not required in the router on the maintenance of the multicast group state, so the core network function is guaranteed, and network can support a large number of multicast groups.

Overlay multicast in order to solve the problem of IP multicast which provides a new means, but also put in a few problems.

\section{A. Data Transmission Routing Performance Problem}

Overlay multicast network is virtual logic network in the IP network. In the IP layer may be formed on repetitive grouping and repeat path, which results in overlay multicast routing path of transmission delay and increased bandwidth. Typically, overlay multicast performance is poor than IP multicast, the problem is even more serious in the multimedia application

\section{B. End the System Dynamic Problem}

With the development of network technology, network is more and more apparently heterogeneous environment. Heterogeneity is mainly manifested in three aspects in overlay multicast. First, it is manifested in the physical network heterogeneity. For example, asymmetric network link delay and different network bandwidth. Second, it is shown as end system which is heterogeneous, for example, different end treatment capacity of the system, different input bandwidth, different output bandwidth, and so on. Third, it reflects the heterogeneous QoS of application. Three of these heterogeneous dramatically increase the overlay multicast routing control difficulty.

\section{Control Overhead}

Control cost refers to the overlay multicast routing algorithm and routing protocol in order to construct and maintain routing required time cost and space cost such as routing protocol state maintenance cost, the algorithm's time complexity and space complexity. One of the key performance indicators for a routing algorithm or protocol is the control overhead, so the balance between the routing performance and control overhead should be mainly considered in the multicast routing algorithms. 


\section{Multicast Routing Algorithms}

A lot of overlay multicast routing protocol and algorithm has been put forward. Overlay multicast routing protocol and algorithm involves technology is very extensive. The algorithm can be classified and constructed in different standard.

\section{A. According to the Structural Approach}

Multicast routing algorithm based on structure can be divided into two types: grid priority and tree priority. Grid priority mode is composed of end system to form a mesh topology, and then based on the grid to construct the multicast tree. Classical routing algorithm method has CoolStreaming [1] and GnuStream [2]. In CoolStreaming, node obtained from the video block adjacent to the point of pull up the video block, the system has robustness. Its disadvantage is the unpredictable video distribution efficiency, which origins from dynamic equivalence relation, this lead to the decline in the video service quality. GnuStream allocates one or several sending nodes as the backup to restore a streaming sessions for a transmitting node failure. Its disadvantage is the larger relative delay. While the tree priority mode is composed of end system to directly form a multicast tree, and non-relies on the network. Classical routing algorithms include ESM [3] and P2PCast [4], based on ESM tree P2P stream media system, allows a user to broadcast audio / video data, any node gets from only one source of data, and supports different quality media, using the node to push data to the child to distribute video. One of the main disadvantages of ESM is the overlay tree led to leave very sensitive system node; node leave can interrupt to the node as the root of all nodes in the subtree of the video distribution. P2PCast can effectively realize the node load balancing. The streaming media is divided into a number of fast methods to balance the user contribution from the full bandwidth, core is to use multicast data is divided into several blocks, then the number of blocks in separate multicast tree propagation. Advantages of greatly enhance the network scalability and fault tolerance.

\section{B. According to Control Approach}

According to the multicast routing algorithm and routing protocol control mode, it can be divided into centralized mode, distributed mode and hierarchical mode. Centralized mode is composed of control center is responsible for overlay multicast network state information collection and routing calculation. Distributed mode is that the state information and the algorithm for distribution to each end system end system node to node, since the organization construction of overlay multicast routing. Hierarchical mode is adopted to construct the multi-level decomposition of multicast routing, the complexity of the control.

\section{1) Centralized Mode}

A typical centralized routing algorithm is the ALMI [5], which monitor and control the multicast group members through the session controller; every member node detects itself to the neighbor node state information, and gives the feedback to the session controller; a session controller according to the obtained information to construct the multicast routing. ALMI algorithm through the limits of each node to a neighboring node degree, maintenance of local topological structure of the network to reduce the control overhead, while using the minimum spanning tree algorithm to construct the degree constrained minimum cost multicast tree sharing, the protocol is a centralized manner to maintain the state of the whole system information, cause the system can poor expansibility.

\section{2) Distributed Mode}

Typical distributed routing algorithms include Narada [6], Overcast [7] and Banerjee [8]. Narada algorithm uses distributed multicast group management, all members of a multicast group constitutes a virtual network topology. When a new node is to be added to the network, the node can get a group member list, in which random choice of its neighbor nodes to request to join the network topology, and using a reverse algorithm of the shortest path to the data forwarding. The protocol has many advantages. For example, pair of nodes and edges in the state is dynamically updated, easily realization of multicast tree routing mechanism, and meeting the different service applications, adopting corresponding parameters to construct the multicast routing. But it also has some disadvantages, for example, every cycle to maintain the multicast group communication overhead for the $\mathrm{O}\left(\mathrm{n}^{2)}\right.$, so it can only be applied in middle and small scale multicast.

The Overcast protocol is an overlay network multicast system. To maximize the average bandwidth from the source node to the destination node, it uses a tree multicast protocol to maintain the information of the node. When a new node is added to the network, the first connection to the multicast tree root node, then use the dynamic optimization strategy adjustment of the nodes in the multicast tree location, and regularly on the multicast tree optimization. Disadvantage of the method is the data layer and the state level using the tree multicast protocol repeatedly results in the drop of system performance and the fault tolerance. Research on Algorithm of Banerjee based on the proxy overlay multicast system routing protocols, and puts forward two kinds of NP (NonDeterministic Polynomial) hard problem: the degree constrained minimum spanning tree and the average delay of the degree constrained minimum spanning tree delay radius. Banerjee algorithm is a distributed routing algorithm, using a centralized approximation optimization algorithm, according to the node to the source node delay of their rank, then added to the tree, then the nodes periodically locally topological structure adjustment and optimization. The disadvantage of which is that it can only maintain the local state.

\section{3) Hierarchical Mode}

Hierarchy is multicast routing algorithm, which is a typical representative of the Nice [9] and Zigzag [10]. Nice, a host based multicast application, is a self-organized, extended overlay multicast routing protocol in the application layer. It has many stream media applications for low-bandwidth environment. The main idea for the protocol is to constitute a hierarchical structure, which is composed by multicast hosts. Each cluster has a chief cluster, and the chief cluster has the minimum distance to the other hosts. The chief cluster detects the availability by exchanging heartbeat messages periodically. Its advantages are based on 
the application layer multicast, constructed a very low overhead hierarchical control structure, with very little cost to build and maintain an application layer multicast tree. Its disadvantage is the hierarchical structure of the robustness is bad; the cluster head dependence is very strong, and only suitable for low bandwidth, with a large number of the receiver set of streaming media application. Nice always uses a cluster head to submit content to the clustering of other members of the organization, and Zigzag receiver into a clustering hierarchy, and according to the rules of C-rules in this hierarchy structure to build multicast tree. In the cluster, a cluster is a cluster head and deputy head of cluster, the cluster head in charge of overseeing the clustering of all members of the cluster head, side responsible for the transmission of content to the clustering of other members. Cluster head failure will not affect other cluster members of the service continuity, when cluster head left, cluster head still work, and be able to quickly specify a pair of cluster head. So the search performance is concerned, node structure maintenance is harsh, and is hard to realize that there is a certain degree of difficulty.

\section{According to Optimization Objective and Constraint Condition}

Solving the overlay multicast routing problem is the main target of optimization and conditions. The optimization target is divided into two kinds: node optimization and the optimal tree, the constraints are divided into two kinds: node constraint and the tree constraint. The optimization of node and node constraint is directed to nodes associated attributes, such as node bandwidth, cost, degree, and the optimal tree and tree constraint for multicast tree related properties, such as tree delay radius, average delay, and cost. The optimization objectives and constraint conditions are combined, which can form a variety of types of overlay multicast algorithm. Multicast routing algorithms based on optimization include RT [11] and dHCPS [12] in the P2P real-time stream media system. RT algorithm of multicast tree session in the covering map overlays the optimization. It's essence in the multicast tree by sending a probe message to reverse detection from the receiving node to the sending node of the optimal path, thereby reducing the delay of multicast tree. The algorithm cannot improve the performance of streaming media system effectively. The detecting information within the nodes sent to their neighbors randomly, this may result in the minimum delay path is not chose as the course of covering graph optimization, so the optimization between covering graphs can not accomplish completely. RT can not effectively improve the performance of streaming media system. dHCPS algorithm in hierarchical clustering structure adopts a company describing the system maximum flow rate and the actual media. The robustness system of dHCPS is poor, the cluster head dependence is too large, and if the cluster head failure which lead to the root of the subtree cannot receive distributed video.

\section{CONCLUSIONS}

To improve the quality of services and utilize the cyber sources effectively, the overlay routing algorithms need to search an optimal path or a satisfying path. The algorithm performance can be evaluated by three indicators. The first one is the degree of stretching, it represents the extra routing. The second one is the stress, it means the extra bandwidth in overlay multicast routing. The third one is the cost; it represents the utilization of network resource in a multicast routing. An excellent algorithm means that it would show good performance in all the three aspects. But in actual applications, the three mentioned indicators may conflict, and the multimedia application has different service requirements in bandwidth, delay and delay jitter. The algorithm designer should prefer parts of the three indicators by different requirements. The designer should consider the generality, expansibility and simplicity also. P2P streaming media technology is widely used in wireless environment now, the demand for flow application is becoming greater and greater. This makes the problem of P2P multicast routing is more complex in wireless network. More efforts should be paid to solve these problems.

\section{REFERENCES}

[1] X. Zhang, J. Liu, B. Li, and T. Yum, "CoolStreaming/DONet: A datadriven overlay network for live media streaming," Proc. IEEE infocom, IEEE Press, 2005, pp. 2102-2111.

[2] X. Jiang, Y. Dong, D. Xu, and B. Bhargava, “GnuStream: a P2P media streaming system prototype,” Proc. IEEE Conf Multimedia and Expo(ICME), IEEE Press, vol. 1, 2003, 325-328.

[3] Carnegie Mellon University. End System Multicast. http:// esm.cs.cmu.edu,2005.

[4] A. Nicolosi and S. Annapureddy, "P2PCast: A peer-to-peer multicast scheme for streaming data,” Proc. IRIS Student Workshop, Cambridge, MA, UK, August 2003.

[5] D. Pendarakis, S. Shi, D. Verma, and M. Waldvogal, "ALMI:an application level multicast infrastructure,” Proc. 3rd USITS, 2001, pp.49-60.

[6] Y. Chu, S. Rao, H. Zhang. “A Case for End System Multicast,” Proc. ACM SIGMETRIC 2000. Santa Clara, California: ACM, 2000, pp.112.

[7] J. Jannotti, D. Gifford, K. Johnson, “Overcast: Reliable Multicasting with an Overlay Network,” Proc. infocom, IEEE Press, 2000, pp. 1114.

[8] R. Lyengar, S. Banerjee, "Low-Coordination Topologies for Redundancy in Sensor Networks,” Proc. IEEE infocom, IEEE Press, 2005, pp.332-342.

[9] Banerjee S, Bhattacharjee B, Kommareddy C. "Scalable application layer multicast,” Proc. ACM SIGMETRIC 2002. Pennsylvania, ACM, 2002, pp. 205-217.

[10] D. A. Tran, K. A. Hua, and T. T. Do, "A peer-to-peer architecture for media streaming,” IEEE J. Selected Areas in Comm.(JSAC), vol. 22, no. 1, 2004, pp.121-133.

[11] X. Liao, H. Jin, Y. Liu, L. Ni, and D. Deng, “Anysee: Peer-to-Peer live streaming,” Proc. IEEE Infocom, IEEE Press, 2006, pp.1-10.

[12] G. Yang, L. Chao, L.Yong, "Decentralized Hierarchically Clustered P2P Video Streaming,” Proc. ACM Sigmetrics, ACM Press, 2008, pp.655-622. 